\title{
RELATIONS BETWEEN THE OPTICAL AND RADIO PROPERTIES OF EXTRAGALACTIC RADIO SOURCES
}

\author{
E. M. Burbidge \\ University of California, San Diego
}

\section{INTRODUCTION}

Correlations between the radio and optical properties of radio sources have proved elusive and the main conclusion to be drawn from this is that there is a great variety of objects in the universe that emit nonthermal radiation, so that attempts to use these objects for cosmological purposes can be frustrated unless one can find some way of selecting objects that do have common intrinsic properties. Despite this, the search for relations and correlations is interesting quite apart from cosmology, because such correlations should provide a groundwork for a physical theory or theories of what is really happening in sources of nonthermal radiation.

I want first briefly to discuss radio galaxies, because the problems here are better definable than for the QSOs, for which the basics, even distances and redshifts, and the very obvious heterogeneity of such objects, pose very great problems in their understanding. Then I shall turn to the QSOs and present some new results.

\section{RADIO GALAXIES}

A recent catalogue of $3 \mathrm{C} R$ sources (Smith, Spinrad, and Smith 1976) lists 137 radio galaxies, and of those for which optical spectroscopic characteristics are given 45 have strong emission lines, 23 have emission lines of moderate strength, 12 have weak emission and 23 have only absorption lines. Thus there appears to be some correlation between a radio source being strong enough to be listed in the $3 C R$ catalogue and the presence in its optical spectrum of emission lines other than just weak [O II] or $\mathrm{H} \alpha$. On the other hand, when one considers galaxies that are weaker radio sources, the situation changes. Data compiled a year ago (Burbidge 1975) for 222 galaxies 
showed that 152 had no strong emission lines in their spectra, while 70 did have strong emission lines. Weaker radio galaxies include ellipticals and spirals with nuclear emission. Nuclear emission from spirals becomes quite common at a low level. The E galaxies contribute heavily to the objects that have only absorption lines in their optical spectra while spirals may have emission lines of weak or average strength, as well as the fewer cases that have strong emission lines.

The range of morphological types of radio galaxies have changed little since the original categorization by Matthews, Morgan, and Schmidt (1964). There are $15 \mathrm{~N}$ systems in the 3CR set of 137 galaxies. It is worth remembering that the original definition by Morgan (1958) for the N-type galaxies is "systems having small, brilliant nuclei, superposed on a considerably fainter background." The "db" or dumbbell galaxies, i.e. double ellipticals in a common stellar envelope, have however been found to be much rarer than appeared in the Matthews, Morgan, and Schmidt list. The giant E or $\mathrm{D}$ galaxies, very often the brightest members of clusters of galaxies, still predominate.

Several papers in this symposium have discussed correlations between radio spectral index and luminosity of radio galaxies, and the paper by Osterbrock describes in detail optical spectrophotometric results for a number of radio galaxies. I think one can say that there is a tendency for a compact radio or optical object to possess strong emission lines in its optical spectrum. There is especially a tendency for objects with complex radio spectra to be associated with compact optical objects; $\mathrm{N}$ systems certainly fall into this category. However, there is one notable exception to the statement that compactness goes with the presence of strong emission lines, and that is the BL Lac objects. I will discuss these in Section $\mathrm{V}$ of this paper.

\section{QSOs -- GENERAL CONSIDERATIONS}

One of the important questions which one would like to be able to answer is "What is the relation between radio-emitting and radioquiet QSOs?" QSOs were first discovered as radio sources, but apparently they are far outnumbered in the sky by the radio-quiet objects. We have estimates from Sandage and Luyten (1967) of the number of radio-quiet QSOs per square degree down to a magnitude limit of 19.7; the number usually adopted is 5 per square degree. This number may, however, need revision; it may not be simply extendable to fainter objects; it may even not be constant across the sky. These are all questions which need to be looked into and new 
programs for finding radio-quiet QSOs from objective prism spectra, both by $M$. Smith and P. Osmer in the southern hemisphere and by A. A. Hoag in the northern hemisphere, are expected to throw some light on this question soon. Harding E. Smith, A. A. Hoag and I have been observing some of the objects in the region of sky covered by the Hercules cluster of galaxies; these objects were discovered by A. A. Hoag in his objective "grism" study using the 4-meter telescope at Kitt Peak. The preliminary results are very interesting, since there appear to be a large number of objects - - 16 candidate QSOs in the first field of approximately one square degree which was searched, of which 8 have been confirmed, and something like 11 candidates in the second field nearby but off the Hercules cluster. This search method is subject to observational selection in that objects of high redshift will predominantly be found, since they show Lya emission which is the strongest line. We need to extend such research to objects of lower redshift. The old way of finding radio-quiet QSOs, by looking for objects with UV excess, may still be the best way of finding low-redshift objects, and, since this method may discriminate against high-redshift objects, a combination of the two may cover the whole redshift range.

Let me now turn to some comments on the models for QSOs in which photoionization is the input that controls the region giving rise to the emission lines. One important question is what happens at the Lyman limit; this can be observed in objects at high redshift. Among such high-z objects there is a range from those in which there is no discernible discontinuity at the Lyman limit to those in which there is a very heavy and almost complete absorption there so that very little radiation is emitted immediately shortward of $912 \AA$. This emphasizes once again the gross differences that exist from one object to another. The emission-line spectrum in any one object also clearly indicates that there are inhomogeneities of temperature and density in the region producing the emission lines. In addition, the line-emitting material must be concentrated in blobs which do not fully cover the continuum source, for the following reason. The ratio of Lya to Ly $\beta$ emission in those objects of sufficiently high redshift to show $L y \beta$ is such that Ly $\alpha$ is very strong and Ly $\beta$ is weak or almost absent. This can be interpreted as being due to an optically thick line-emitting region. Every Ly $\alpha$ photon which is produced throughout the emission region escapes after many scatterings. But Ly $\beta$ photons a re degraded into $\mathrm{Ly}_{\alpha}+\mathrm{H}_{\alpha}$ and thus the $\mathrm{Ly}_{\beta} \beta$ that one sees comes only from a thin skin of the emitting region. But optically thick material must not fully cover the continuum source because it would then cause a very large absorption at the Lyman limit, and as already noted there are objects in which this is not the case. Thus the line-emitting 
material may be distributed in blobs which partially cover the continuum source. Another possible model is one in which the emitting gas does not have spherical symmetry about the central object. Indeed, this might well be the case if the central object is a strongly gravitating collapsed mass in which there was initial angular momentum.

In connection with models, before describing some specific results that I would like to present, I want to mention the absorptionline spectra that appear at many redshifts in objects of high $z$. It appears promising to explain these by means of radiation-pressuredriven outflow of gas, which can be accelerated to high velocity and is then subject to instabilities so that it breaks up into small filaments. A new consideration is that the outflow might also be driven by cosmic ray pressure. Some work along these lines is being done at the Max Planck Institute for Astrophysics in Munich, in collaboration with G. Burbidge.

Another question to consider is whether or not the apparent abrupt drop in the redshift distribution at $z \gtrsim 2.3$ is real. It is not clear to what extent this may be due to observational selection. If objects are identified with radio sources on the basis of their having a UV-excess or appearing as blue stellar objects on the Palomar Atlas, there might very well be such a selection against high-z QSOs, because some of those objects have heavy absorption beyond their Lyman limit and some have a multitude of absorption lines shortward of Ly $\alpha$, so they will not appear strong in the ultraviolet and therefore may well be missed. The new searches designed to pick up highredshift quasars should provide new statistics here.

\section{QSOs - - OBSERVATIONAL RESULTS}

Before describing these, let me outline ways in which optical astronomers can work together with radio astronomers:

1. One can work with a well-defined radio sample and get optical observations of as many objects as possible at low signal to noise.

2. One can work on particular, limited, radio samples, for example, those selected by means of their radio spectral properties.

3. One can work on individual objects which have been selected either from their radio or optical properties as being of particular interest. 
Programs underway include examples of all three.

\section{Optical Survey of Molonglo Radio Sources}

Identifications and optical observations of sources in the MC2 and MC 3 catalogues have been made by a group including C. Hazard, $H$. Murdoch, J. Baldwin, H. E. Smith, E. J. Wampler, and myself. The optical observations were made at Lick Observatory with the $3-\mathrm{m}$ telescope and the Wampler-Robinson image dissector scanning spectrograph. The $+11^{\circ}$ and $+16^{\circ}$ of the $\mathrm{MC} 2 / 3$ from R. A. $111 / 2$ to $17 \mathrm{~h}$ have been fairly well covered. There are 65 candidate QSO identifications, of which 42 have had reliable spectroscopic observations. Of the BSOs, 38 proved to be QSOs and 4 were galactic stars. This indicates a high degree of reliability, around $90 \%$, for the identifications. Work in other regions of the sky covered by the MC2 and MC 3 catalogue is less advanced, but a similar ratio of reliability of identification has been found in the R. A. 20 to $24 \mathrm{~h} \mathrm{region.} \mathrm{For} \mathrm{the}$ whole two strips, we have so far 57 confirmed QSOs and $2 \mathrm{BL}$ Lac objects. In addition to BSOs, several red stellar objects which might be identifications of the radio sources were observed, in an attempt to be sure not to discriminate against high-z QSOs. Of these, 9 proved to be galactic stars and 1 was one of the 2 BL Lac objects found. This strongly suggests that in this sample there do not exist a large number of very high redshift red or neutral color objects. There are 8 objects which have continuous spectra, i. e. our observations have sufficiently high signal to noise to indicate that these are not galactic stars and may well be candidate BL Lac objects.

Of the 600 known QSOs, this Molonglo sample comprises $10 \%$. It is of interest to consider properties such as the mean redshift and distribution of redshift. We find $\langle z\rangle=1.162$, which is very similar to $\langle z\rangle$ found for the $4 \mathrm{C}$ QSOs. The distribution in redshift is also similar to that found from previous samples and again indicates a paucity of objects with redshift $z>2.2$. It is interesting that there is a suggestion of a peak at a redshift of about 0.8 to 0.9 , which does not appear in the distribution for the total 600 known QSOs. There are of course not enough observations so far to say whether this is a real peak.

We have looked into the question whether there is a systematic variation of redshift a round the sky at a level corresponding to $10 \%$ in velocity or variation in numbers of QSOs around the sky. There may be some indications of patchiness, but it is too early to say anything definite one way or the other. 
With this good sample of objects, it is interesting to look for a correlation of the continuum spectral index with important emission lines such as $\mathrm{Ly} \alpha, \mathrm{C} \mathrm{IV}, \mathrm{Mg}$ II, and $\mathrm{H} \beta$. In a preliminary search we found no correlation, although the simple photoionization model would predict a dependence of equivalent width of emission lines upon the shape of the optical continuum. I believe the only conclusion to be drawn from this is again that the QSOs comprise objects which have a very wide range of physical conditions.

I want to turn now to very preliminary results by the same group of observers on sources in the Molonglo faint survey, MC5, using identifications by D. Jauncey, C. Hazard and H. Murdoch. H. E. Smith and I obtained spectra of some half dozen objects and found the first four to be high-z QSOs:

\begin{tabular}{cc|cc} 
Object & Redshift & Object & Redshift \\
\hline $0758+120$ & 2.66 & $0830+115$ & 2.97 \\
$0824+110$ & 2.29 & $0938+119$ & 3.19
\end{tabular}

Again, a wide range of physical conditions must be present in these four objects. $0938+119$ and $0830+115$ are like the Ohio $\mathrm{cm}$-excess objects $O Q 172$ and $O H 471$ which have flat or peaked radio spectra. In $0938+119$ there is a clear discontinuity near the Lyman limit but as in $\mathrm{OH} 471$ this does not occur at the wavelength corresponding to $\mathrm{z}_{\text {em }}$ but at a shorter wavelength, and it is probably produced by a multitude of absorption lines. $0830+115$, like OQ 172 , does not have a Lyman discontinuity but has hundreds of narrow absorption lines shortward of Ly $\alpha$ emission. It is interesting to note that none of the 5 QSOs with highest $\mathrm{z}$ (OQ 172, OH 471, 0938+119,0830+115, and $4 \mathrm{C} 5.34$ ) have complete absorption in the Lyman continuum continuum at a wavelength corresponding to that given by their emission-line redshift system. Yet the absence of Ly $\beta$ emission again indicates that the regions producing the emission lines is optically thick as discussed above.

Concerning the emission-line strength in these high-z objects, the ratio C IV to $\mathrm{Ly}_{\alpha}$ is larger than the "normal" value of $1 / 4$. O VI is present in $0830+115$ and $0938+119$.

\section{Red QSOs and BL Lac Objects}

I want now to turn to consideration of two groups of objects, those which I shall call the red QSOs, and the BL Lac objects. There are six $3 \mathrm{C}$ objects which have been identified as QSOs, but which are distinctly red in color: 

$3 \mathrm{C} 68.1$
$3 \mathrm{C} 154$
3C 418
$3 \mathrm{C} 108$
$3 \mathrm{C} 212$
3 C 422

Boksenberg showed that $3 \mathrm{C} 68.1$ has a very steep slope in its optical continuum with $\alpha \approx 6$. These objects are quite difficult to observe because they tend to be faint. Harding E. Smith has studied 3C 418 . It has a $\mathrm{m}_{\mathrm{v}} \approx 21.0$, and a continuum slope in the optical region $\alpha=4$. 3. This value of 4.3 may possibly be reduced to about 4 if one corrects for an average extinction in our own Galaxy. The redshift $z=1.687$. It has Mg II, C III], and C IV emission lines of normal strength. That these objects are fairly rare is indicated by the fact that only these six have emerged in the approximately 50 QSOs in the 3C catalogue. However, it must be remembered that due to their red color and their faintness they may have been discriminated against in identification and their actual relative numbers may be higher than indicated by these results.

The BL Lac objects are extremely compact in both their radio and optical radiating regions, as is indicated by the characteristic features by which one defines BL Lac objects - - that they show very strong variability on very short time scales, and a high degree of polarization which itself is variable in both extent and angle. What distinguishes the BL Lac objects from the optically violent variable QSOs is the lack of reasonably strong emission lines in them. They were first categorized as having no emission lines at all, but some, when high $\mathrm{S} / \mathrm{N}$ observations were obtained, have been shown to have weak emission lines. The lack of strong emission lines can be due to two causes -- either a lack of ionizing radiation, or a lack of gas to be ionized. The former is suggested by the fact that the BL Lac objects resemble the red QSOs in having steep spectral indices and they appear neutral or reddish in color on the Sky Survey plates. One may note that there is plenty of gas seen in the spectrum of one very interesting BL Lac object, viz. AO 0235+164. This absorbing gas was shown by Burbidge et al. (1976) and Rieke et al. (1976) to possess

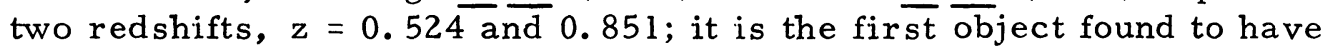
two absorption-line redshifts less than $z=1$.

If one attempts to explain the weakness of the emission lines in BL Lac objects as being due to steepness of the optical continuum, however, it is interesting to note that the C IV line is quite strong in the red QSO $3 C 418$, and it requires very short- $\lambda$ radiation to produce $\mathrm{C}^{+++}$. This argues for there being a considerable flux of either highenergy particles or shock waves to ionize the gas, or else that the continuum is complex and increases again at shorter wavelengths than can be observed from the ground. 
V. THE RADIO SOURCE 3C 303

$3 C 303$ has a very interesting history and is an example of my third category of cooperation between radio and optical observers. Wyndham (1966) identified the source as an N-type galaxy, but noted that there was a blue stellar object about $20^{\prime \prime}$ away which might also be the source. Sandage (1973) measured $z=0.141$ for the galaxy, from strong emission lines. Later observations with higher resolution indicated that the radio source was complex and encompassed both the galaxy and the BSO. Optical observations using an electronographic camera were made by Wlérick and Lelièvre (1975), who noted that there was actually a group of three objects within a few seconds of arc which all had a strong UV excess. These lay within the complex radio source and appeared to be connected with it. The brightest of the group of UV excess objects was quite faint, being a round 20th magnitude, and at the time my attention was drawn to $3 \mathrm{C} 303$, by $P$. Véron, it was not possible to observe with a conventional spectrograph because of sky background. It was necessary to wait until the WamplerRobinson image dissector scanner was available on the Lick $3-\mathrm{m}$ telescope, so that sky subtraction could be performed.

Kronberg has made detailed radio observations at NRAO and Westbork and has mapped the structure of the radio source and has noted that the optical object, the $\mathrm{N}$ galaxy and the group of UV-excess objects lie, as described by the French workers earlier, within the radio source and seem to be associated with its strongest isophotes. One of the fainter objects lies right on the compact center of the western component of the radio source. Thus, before optical observations were made, it appeared that all the optical objects were likely to be physically associated. The brightest of the UV excess objects, according to Wlérick and Lelièvre, has $B=20.05, B-V=0.08$, $U-B=-0.59$. After two seasons of observation, H. E. Smith and I have found that this object is indeed a QSO, with a redshift $z=1.57$ from lines of $\mathrm{Mg}$ II, C III], and C IV, whereas the galaxy has a redshift less than $10 \%$ of this. Accepting the evidence from the radio structure as indicating that the radio source is one single source with complex parts, then we have indeed what I would call the best case I know of a QSO physically associated, it appears, with a galaxy of much lower redshift. Now there is of course always the possibility that the QSO is a radio-quiet object which happens by chance to fall within the radio contours and only appears to be connected with them. And this points up again the necessity to find out just what is the surface density of radio-quiet QSOs across the sky. 
Nevertheless, the coincidence of the close grouping of $3 \mathrm{UV}$ excess objects within a few seconds of arc of each other, and one of these being a QSO, is very striking. All three lie less than $20^{\prime \prime}$ from the $\mathrm{N}$ system. It is, of course, very desirable that the other two UVexcess objects be observed spectroscopically. Unfortunately, they are $11 / 2$ - 2 mag. fainter than the QSO. However, we shall attempt to observe the object that coincides with the compact radio core.

\section{REFERENCES}

Burbidge, E. M.: 1975, Lectures in Radio Galaxies (Urbino Summer School, ed. G. Setti, D. Reidel Publ. Co., Dordrecht-Holland).

Burbidge, E. M., Caldwell, R. D., Smith, H. E., Liebert, J., and Spinrad, H.: 1976, Astrophys. J. Letters 205, L117.

Lelièvre, G. and Wlérick, G. : 1975, Astron. and Astrophys. 42, 293. Matthews, T. A., Morgan, W. W., and Schmidt, M.: 1964, Astrophys. J. 140, 35 .

Morgan, W. W.: 1958, Pub. Astron. Soc. Pacific 70, 364.

Rieke, G. H., Grasdalen, G. L., Kinman, T. D., Hintzen, P., Wills, B. J., and Wills, D.: 1976, Nature 260, 754.

Sandage, A.: 1973, Astrophys. J. 180, 687.

Sandage, A. and Luyten, W. J.: 1967, Astrophys. J. 148, 767.

Smith, H. E., Spinrad, H., and Smith, E. O.: 1976, Pub. Astron. Soc. Pacific, in press.

Wyndham, J.: 1966, Astrophys. J. 144, 459.

DISCUSSION

Bahcall: Is there a concentration of the red QSOs towards the galactic plane?

E.M. Burbidge: There is no obvious concentration near the plane, and so I do not believe that we can account for these objects by saying that they are reddened by galactic absorption. 
Walsh: Does the proportion of QSOs that are red increase near the Sky Survey print limit? Identifications down to about 19.5 magnitude based on accurate radio positions show very few such objects.

E.M. Burbidge: The objects that I have listed are fairly faint and it may be that there are more of them than has been suspected. Observations of neutral or red identifications for compact radio sources will, I hope, lead to a better idea of their frequency and apparent magnitude distribution.

Kronberg: I have mapped 3C 303 at 3.7 and $11.1 \mathrm{~cm}$ (resolution $\sim 2.2$ and $\sim 7 "$ respectively) with the NRAO interferometer, and more recently at $\lambda 6 \mathrm{~cm}$ ( 7" resolution) at Westerbork together with R.G. Strom. An accurate radio-optical comparison using two deep Palomar 48-inch plates (taken by $S$. van den Bergh) enabled us to show that the cores of both main radio components lie within 1 " of two objects which are 16" arc apart (Kronberg, Ap.J. (Lett) 203, L47, 1976). One of these is a 17th magnitude $\mathrm{N}$-galaxy $(\mathrm{z}=0.14)$ and the other a 22nd magnitude member of a trio of faint objects. Lelièvre and Wlérick (Astron. and Astrophys. $42,293,1975)$ who had independently identified the field have found that all three faint objects $\left(M_{\mathrm{y}}=21.70,19197\right.$ and 21.75) have $\mathrm{UV}$ excess. The radio maps at $11 \mathrm{~cm}$ and $6 \mathrm{~cm}$ show further extended structure on both sides of the N-galaxy, and confirm the presence of faint radio structure between the two main radio components. A radio extension to the southwest of the western radio component includes the entire trio of $\mathrm{UV}$ excess objects. It is the brightest whose redshift $(z=1.57)$ has recently been determined.

The fact of the double radio-optical coincidence is curious. Our radio maps show with virtual certainty that $3 C 303$ is a single radio system, and not an accidental super-position of unrelated radio sources. The question of whether the two systems of different redshift are physically related or not relies on whether the $20^{\mathrm{m}}$ quasar and its two fainter companions coincide only by chance with the western radio component of $3 \mathrm{C} 303$. In any case the optical field is interesting, and the result underlines the need to do such radio-optical comparisons for more sources. We need more deep optical plates and spectra, now that we have detailed multi-frequency radio maps at optical resolution.

Webster: At first glance this looks to be a fairly standard type of radio source with 2 outer steep-spectrum lobes and one central flat spectrum component centred on the bright galaxy. Then this seems to me to be a good case for associating the whole radio source with the galaxy, but what is there to link the quasar with the preceding radio lobe?

Kronberg: The quasar with measured redshift lies approximately in the centre of the southwest extension of the main western radio component, although not on a compact (< l") radio core. One of its fainter (also UV excess) companions does coincide within $\sim 1 "$ with the bright radio core of the tadpole-like western component. 
P. Véron: Lelièvre and Wlérick (Astron. and Astrophys. 42, 293, 1975) have measured the colors of the faint object which coincides with the

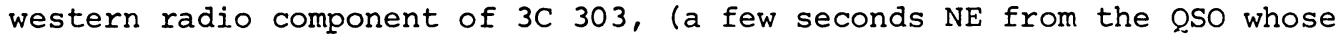
redshift was measured by $M$. Burbidge). This very faint object $(V=21.7)$ has a strong UV excess with $U-B=-0.90 \pm 0.25$, so it is a good quasar candidate. The positional agreement is also very close, since it lies within 1 arc second of the mean Cambridge radio position as determined by Branson et $a l$. and by Pooley and Henbest.

Ryle: Concerning the discussion about 3C 303; I looked back at the map published by Pooley \& Henbest in 1974. This was made with the $5 \mathrm{~km}$ telescope at $\lambda=6 \mathrm{~cm}$ with an angular resolution of 2 " arc, and showed an unresolved $E$ component $<1 " .2$ and $a$ W component $4 " .4 \times 1 " .3$, with a strong polarisation in the northerly extension. These early observations also suggested a faint and extensive envelope and showed no feature associated with the large redshift blue object.

In order to examine the low $\mathrm{T}$ features with better sensitivity we observed the source last night with the $5 \mathrm{~km}$ telescope at $\lambda=11 \mathrm{~cm}$, where the sensitivity for a resolved source is some 10 times better, and the resolution is still 3".7 arc. The map shows a jet running from the compact $E$ source towards the $W$ component, and a faint diffuse extension to the $\mathrm{E}$ of the compact source, whose length is comparable with the separation of the main components. The distribution is reminiscent of those of $3 C 66$ and 3C 219, and suggests that in all these cases the compact source represents the nucleus of a double structure whose components have developed in different ways.

The large redshift blue object does not appear to be associated with any significant radio emission, although the northern blue object lies within the extended $W$ component.

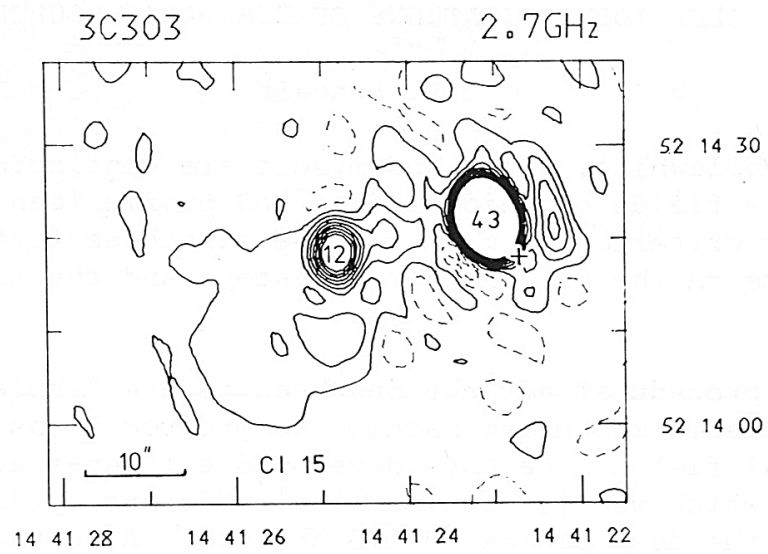

Map of 3C303 made with the $5-\mathrm{km}$ telescope at $2.7 \mathrm{GHz}$. For the two intense components the higher level contours have been omitted and the peak level is indicated. The cross marks the position of the brightest of the three UV excess objects. 
Miller: Is the blue object very near the centre of the western radio component extended, and is it possible to get a spectrogram of it?

E.M. Burbidge: It can be done; it's faint and it's going to take time, but it would be well worth the effort.

Rowan-Robinson: I would like to direct a question to those who identify radio sources with quasars: If there had been only the quasar for which the redshift has been measured and the right hand radio source, would it have been accepted as an identification?

E.M. Burbidge: We would have looked at it as a possible candidate.

Ryle: No, the positional disagreement is far too great and the optical object is not associated with any significant radio emission.

Swarup: For Ooty occultation sources we do not consider any object identified if it is not along the axis of the source or coincident with one component within the error bars.

Hazard: It is doubtful if the oSO near $3 C 303$ would have been identified in the Molonglo survey. The galaxy would have been considered the more probable identification although the QSO would have been noted and examined spectroscopically. It is my opinion that the claimed associations between QSO's and galaxies etc. is a reflection of a very high background gso density per sq degree.

Arp: As someone who has actually observed and searched for ultra violet excess objects on photographic plates - I can testify that such a close configuration of strong ultra violet excess objects as the three near $3 \mathrm{C} 303$ is extraordinary.

\section{DEEP IDENTIFICATIONS OF 3CR RADIO SOURCES}

\section{M.S. Longair}

Gunn, Kibblewhite, Riley and myself are continuing deep optical surveys of the fields of unidentified and poorly identified $3 \mathrm{CR}$ radio sources. Our criterion is $\mathrm{m}>19$. We have been fortunate to obtain observing time on the Palomarg $5-m$ telescope and the Kitt Peak 4-m telescope.

A major procedural advance has been to use Kibblewhite's laser scanning automatic measuring machine to produce scans and contour maps of the optical fields. We have developed a three-stage reduction procedure by which we tie together the radio and optical frames of reference on the deep plates to $0.3 \mathrm{arc} \mathrm{sec.} \mathrm{A} \mathrm{further} \mathrm{advance} \mathrm{is} \mathrm{the}$ possibility of obtaining magnitudes at very faint magnitudes by integrating over the digitised image of the optical counterpart. So far we have studied the first 4 fields of our sample and obtained $100 \%$ success in our identification programme. These sources are 3C133, 184, 190, 217. 3C190 is a relatively bright galaxy and was previously identified but 
the other identifications are believed to be new. In the case of 3 c184 (see figure), the estimated magnitude of the galaxy is $23.2 \pm 0.5$. We have plates of a further 25 fields.

\section{C 184}
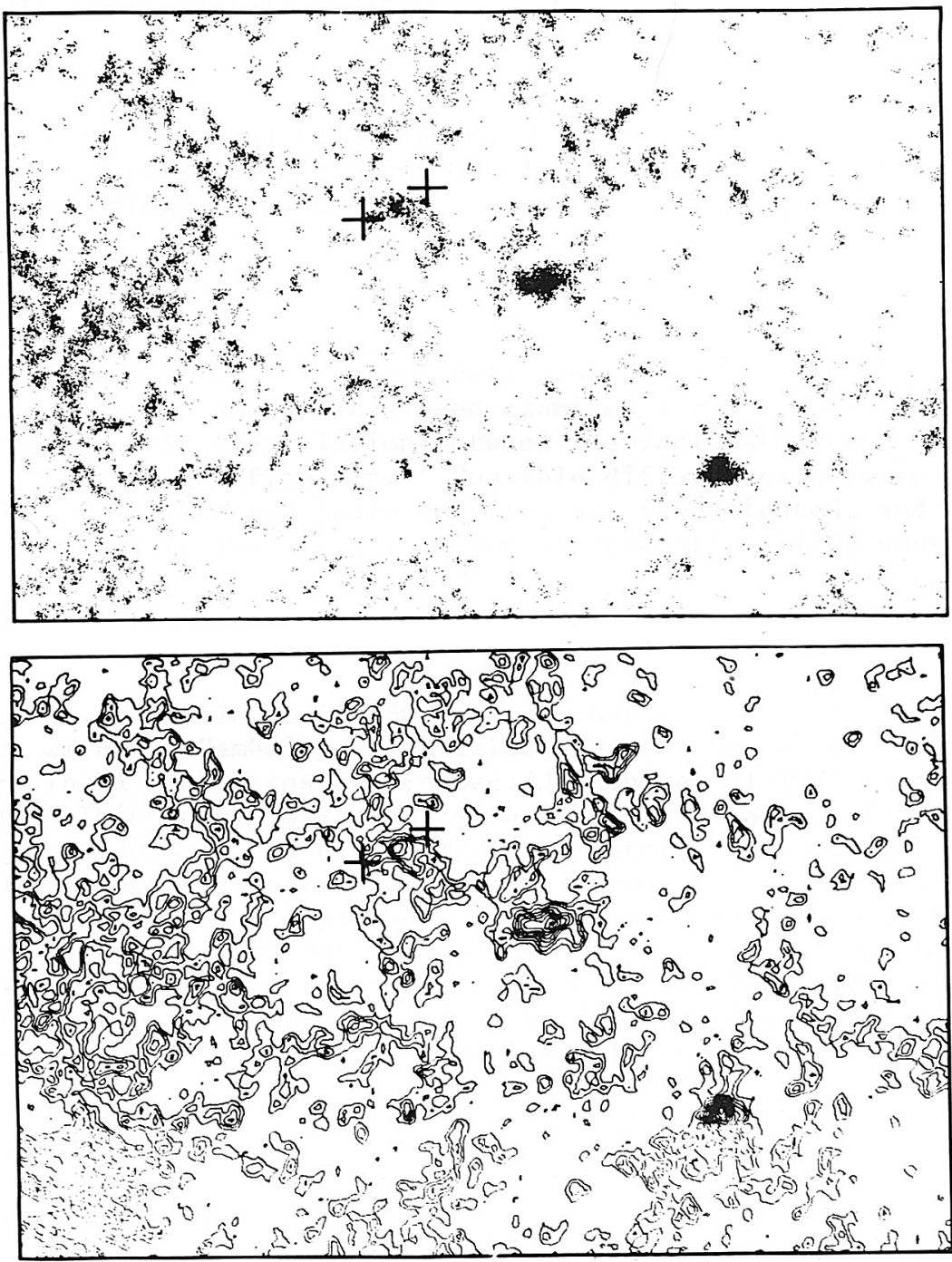

Scans and contour map of the field around 3C 184. The identified galaxy lies between the two crosses. 